\title{
Almost split sequences for non-regular modules
}

\author{
by
}

\author{
Shiping L i u (Singapore)
}

\begin{abstract}
Let $A$ be an Artin algebra and let $0 \rightarrow X \rightarrow \bigoplus_{i=1}^{r} Y_{i} \rightarrow Z \rightarrow 0$ be an almost split sequence of $A$-modules with the $Y_{i}$ indecomposable. Suppose that $X$ has a projective predecessor and $Z$ has an injective successor in the Auslander-Reiten quiver $\Gamma_{A}$ of $A$. Then $r \leq 4$, and $r=4$ implies that one of the $Y_{i}$ is projective-injective. Moreover, if $X \rightarrow \bigoplus_{j=1}^{t} Y_{j}$ is a source map with the $Y_{j}$ indecomposable and $X$ on an oriented cycle in $\Gamma_{A}$, then $t \leq 4$ and at most three of the $Y_{j}$ are not projective. The dual statement for a sink map holds. Finally, if an arrow $X \rightarrow Y$ in $\Gamma_{A}$ with valuation $\left(d, d^{\prime}\right)$ is on an oriented cycle, then $d d^{\prime} \leq 3$.
\end{abstract}

Let $A$ be a fixed Artin algebra, $\bmod A$ the category of finitely generated left $A$-modules and $\operatorname{rad}(\bmod A)$ the Jacobson radical of $\bmod A$. Denote by $\Gamma_{A}$ the Auslander-Reiten quiver of $A$. The shape of a connected component of $\Gamma_{A}$ without projectives or without injectives is fairly well understood [5, $8,12]$. The results of this paper will give some information on connected components of $\Gamma_{A}$ which contain both a projective module and an injective module.

The notion of an almost split sequence, which was introduced by Auslander and Reiten in [1], plays a fundamental role in the representation theory of algebras (see, for example, [10]). Let $0 \rightarrow X \rightarrow \bigoplus_{i=1}^{r} Y_{i} \rightarrow Z \rightarrow 0$ be an almost split sequence in $\bmod A$ with the $Y_{i}$ indecomposable. Then the number $r$ measures the complication of the maps in $\bmod A$ starting with $X$ and those ending with $Z$. Therefore it is interesting to find the number of the indecomposable summands of the middle term of an almost split sequence. The well-known Bautista-Brenner theorem [3] states that if $A$ is of finite representation type, then the middle term of an almost split sequence in $\bmod A$ has at most four indecomposable summands, and the number four occurs only in the case where one indecomposable summand is projective-injective. Our main result clearly generalizes this theorem. Moreover, we will also discuss almost split sequences for modules on oriented cycles in $\Gamma_{A}$.

We begin with the following easy observation. 
Lemma 1. Let $g: Y \rightarrow Z$ be an irreducible epimorphism with $Z$ indecomposable, and let

$$
Z_{n} \rightarrow Z_{n-1} \rightarrow \ldots \rightarrow Z_{1} \rightarrow Z_{0}=Z
$$

be a sectional path in $\Gamma_{A}$ with $n \geq 1$. If there is an irreducible map from $Y \oplus Z_{1}$ to $Z$, then $Z_{i}$ is not projective for $0 \leq i \leq n$ and there is an irreducible epimorphism $g_{i}: D \operatorname{Tr} Z_{i} \rightarrow Z_{i+1}$ for $0 \leq i<n$.

Proof. The lemma follows from the easy facts that if

$$
0 \rightarrow X \stackrel{\left(f, f^{\prime}\right)}{\longrightarrow} Y \oplus Y^{\prime} \stackrel{\left(\begin{array}{l}
g \\
g^{\prime}
\end{array}\right)}{\longrightarrow} Z \rightarrow 0
$$

is an exact sequence, then $g$ is epic if and only if $f^{\prime}$ is epic, and that if $p: M \rightarrow N$ is an epimorphism, then so is the co-restriction of $p$ to a summand of $N$.

We have the following immediate consequence.

Corollary 2. Let

$$
0 \rightarrow X \stackrel{f}{\longrightarrow} \bigoplus_{i=1}^{r} Y_{i} \stackrel{g}{\longrightarrow} Z \rightarrow 0
$$

be an almost split sequence with the $Y_{i}$ indecomposable. If the co-restriction of $f$ to $Y_{i}$ is epic for $1 \leq i \leq r$, then any sectional path in $\Gamma_{A}$ ending with $Z$ contains no projective module.

Proof. Assume that the co-restriction of $f$ to $Y_{i}$ is epic for all $1 \leq i \leq r$. Let

$$
Z_{n} \rightarrow Z_{n-1} \rightarrow \ldots \rightarrow Z_{1} \rightarrow Z_{0}=Z
$$

be a sectional path in $\Gamma_{A}$ with $n>0$. Then $Z_{1} \cong Y_{i_{0}}$ for some $1 \leq i_{0} \leq r$ and $Z_{2} \neq X$ if $n \geq 2$. Now there is an irreducible epimorphism $h: X \rightarrow Z_{1}$ by assumption. Hence $Z_{1}$ is not projective, and if $n>1$, then $Z_{j}$ with $2 \leq j \leq n$ is not projective by Lemma 1 .

We quote the following lemma from [9].

Lemma 3. Let $p: M \rightarrow Y$ be a non-zero map with $Y$ indecomposable, and let $f: Y \rightarrow Z_{1} \oplus Z_{2}$ be an irreducible map with $Z_{1}, Z_{2}$ indecomposable. If $p f=0$, then $Y, Z_{1}, Z_{2}$ are not projective, moreover, there is a map $q$ : $M \rightarrow D \operatorname{Tr} Y$ in $\bmod A$, a map $v: D \operatorname{Tr} Y \rightarrow Y$ in $\operatorname{rad}(\bmod A)$ and a source map

$$
\left(h_{1}, h_{2}, h\right): D \operatorname{Tr} Y \rightarrow D \operatorname{Tr} Z_{1} \oplus D \operatorname{Tr} Z_{2} \oplus U
$$

such that $p=q v$ and $q h=0$.

In the case where there is an irreducible epimorphism $f: P \rightarrow Z$ with $P$ indecomposable projective, Auslander and Reiten described in [1] the almost split sequence ending with $Z$. Thus the following fact is of interest. 
Corollary 4. If $f: P \rightarrow Z$ is an irreducible epimorphism with $P$ indecomposable projective, then $Z$ is indecomposable. Dually, if $g: X \rightarrow I$ is an irreducible monomorphism with I indecomposable injective, then $X$ is indecomposable.

Proof. Assume that $f: P \rightarrow Z$ is an irreducible epimorphism. Let $k: K \rightarrow P$ be the kernel of $f$; then clearly $k f=0$. Thus $Z$ is indecomposable by Lemma 3 .

An indecomposable module $X$ in $\bmod A$ is said to be left stable if $D \operatorname{Tr}^{n} X \neq 0$ for all $n \geq 0$, and right stable if $\operatorname{Tr} D^{n} X \neq 0$ for all $n \geq 0$. Let ${ }_{l} \Gamma_{A}$ be the full subquiver of $\Gamma_{A}$ generated by the left stable modules, and ${ }_{r} \Gamma_{A}$ the full subquiver generated by the right stable modules. We call the connected components of ${ }_{l} \Gamma_{A}$ left stable components of $\Gamma_{A}$, and those of ${ }_{r} \Gamma_{A}$ right stable components of $\Gamma_{A}[8]$.

For a module $M$ in $\bmod A$, we denote by $\ell(M)$ its composition length.

Lemma 5. Let $f: X \rightarrow \bigoplus_{i=1}^{4} Y_{i}$ be an irreducible map with $X$ indecomposable and the $Y_{i}$ indecomposable non-projective. If $f$ is epic or $\ell(X) \geq \ell(\operatorname{Tr} D X)$, then

(1) $X$ has no projective predecessor in $\Gamma_{A}$;

(2) $\ell\left(D \operatorname{Tr}^{n} X\right)$ monotone grows to infinity;

(3) $X$ is not on any oriented cycle in $\Gamma_{A}$.

Proof. Assume that $f$ is epic or $\ell(X) \geq \ell(\operatorname{Tr} D X)$. We claim that $2 \ell(X) \geq \sum_{i=1}^{4} \ell\left(Y_{i}\right)$.

Indeed, this is clear if $f$ is epic. Otherwise $\operatorname{Tr} D X \neq 0$ and $\ell(X) \geq$ $\ell(\operatorname{Tr} D X)$. Hence $2 \ell(X) \geq \ell(X)+\ell(\operatorname{Tr} D X) \geq \sum_{i=1}^{4} \ell\left(Y_{i}\right)$.

Let $h: D \operatorname{Tr} X \rightarrow W$ be an irreducible map with $W$ indecomposable. If $W \neq D \operatorname{Tr} Y_{i}$ for all $1 \leq i \leq 4$, then

$$
\begin{aligned}
\ell(D \operatorname{Tr} X) & \geq \ell(W)+\sum_{i=1}^{4} \ell\left(D \operatorname{Tr} Y_{i}\right)-\ell(X) \\
& \geq \ell(W)+\sum_{i=1}^{4}\left(\ell(X)-\ell\left(Y_{i}\right)\right)-\ell(X)>\ell(W) .
\end{aligned}
$$

If $W \cong D \operatorname{Tr} Y_{i}$ for some $i$, say $W \cong D \operatorname{Tr} Y_{1}$, then

$$
\begin{aligned}
\ell(D \operatorname{Tr} X) & \geq \sum_{i=1}^{4} \ell\left(D \operatorname{Tr} Y_{i}\right)-\ell(X) \\
& \geq \ell(W)+\sum_{i=2}^{4}\left(\ell(X)-\ell\left(Y_{i}\right)\right)-\ell(X) \geq \ell(W) .
\end{aligned}
$$


Thus $h$ is epic. By Corollary 2, any sectional path in $\Gamma_{A}$ ending with $X$ contains no projective module. Moreover, we have

$$
\begin{aligned}
\ell(D \operatorname{Tr} X) & \geq \sum_{i=1}^{4} \ell\left(D \operatorname{Tr} Y_{i}\right)-\ell(X) \\
& \geq \sum_{i=1}^{4}\left(\ell(X)-\ell\left(Y_{i}\right)\right)-\ell(X) \geq \ell(X) .
\end{aligned}
$$

By induction we have $\ell\left(D \operatorname{Tr}^{n+1} X\right) \geq \ell\left(D \operatorname{Tr}^{n} X\right)>0$ for all $n \geq 0$, and any sectional path in $\Gamma_{A}$ ending with $D \operatorname{Tr}^{n} X$ contains no projective module. Thus $X$ has no projective predecessor in $\Gamma_{A}$.

Since $2 \ell(X) \geq \sum_{i=1}^{4} \ell\left(Y_{i}\right)$, either $\ell(X) \geq \ell\left(Y_{1}\right)+\ell\left(Y_{2}\right)$ or $\ell(X) \geq \ell\left(Y_{3}\right)+$ $\ell\left(Y_{4}\right)$. Thus we may assume that the co-restriction $g: X \rightarrow Y_{1} \oplus Y_{2}$ of $f$ is epic. Let $k: K \rightarrow X$ be the kernel of $g$. By Lemma 3, there is a map $k_{1}: K \rightarrow D \operatorname{Tr} X$ in $\bmod A$, a map $v_{1}: D \operatorname{Tr} X \rightarrow X$ in $\operatorname{rad}(\bmod A)$ and an irreducible epimorphism $g_{1}: D \operatorname{Tr} X \rightarrow D \operatorname{Tr} Y_{3} \oplus D \operatorname{Tr} Y_{4}$ such that $k=k_{1} v_{1}$ and $k_{1} g_{1}=0$. By induction, for all $n>0$, there is a map $k_{n}$ : $K \rightarrow D \operatorname{Tr}^{n} X$ and a map $v_{n}: D \operatorname{Tr}^{n} X \rightarrow D \operatorname{Tr}^{n-1} X$ in $\operatorname{rad}(\bmod A)$ such that $k=k_{n} v_{n} \ldots v_{1}$. Hence $\ell\left(D \operatorname{Tr}^{n} X\right)$ tends to infinity by the Harada-Sai lemma [6]. In particular, $X$ is not $D$ Tr-periodic.

Let $\Gamma$ be the left stable component of $\Gamma_{A}$ containing $X$. Then $\Gamma$ contains no $D$ Tr-periodic module since $X$ is not. Note that all predecessors of $X$ in $\Gamma_{A}$ are left stable, hence in $\Gamma$. In particular, the $D \operatorname{Tr} Y_{i}$ are in $\Gamma$. So $\Gamma$ contains no oriented cycle $[8,(2.3)]$. Thus $X$ is not on any oriented cycle in $\Gamma_{A}$. The proof is complete.

We also need the following lemma.

Lemma 6. Let $X$ be an indecomposable module in $\bmod A$ such that there is a sectional path from $X$ to an injective module in $\Gamma_{A}$. Assume that $f: X \rightarrow \bigoplus_{i=1}^{r} Y_{i}$ is a source map with the $Y_{i}$ indecomposable. If $r>4$ or $r=4$ with all $Y_{i}$ non-projective, then $X$ has no projective predecessor and is not on any oriented cycle in $\Gamma_{A}$.

Proof. Let $r \geq 4$, and let

$$
X=X_{0} \rightarrow X_{1} \rightarrow \ldots \rightarrow X_{t-1} \rightarrow X_{t}
$$

be a shortest sectional path in $\Gamma_{A}$ with $X_{t}$ injective. If $t=0$, then $X$ is injective. Therefore $f$ is epic. Thus the lemma holds by Lemma 5 .

Suppose now that $t>0$ and $X_{1} \cong Y_{1}$. Then $X_{j}$ is not injective for $0 \leq j<t$, and there is an irreducible epimorphism $f_{t}: X_{t} \rightarrow \operatorname{Tr} D X_{t-1}$. By Lemma 1 , there is an irreducible epimorphism $f_{1}: Y_{1} \rightarrow \operatorname{Tr} D X$. It follows then that the co-restriction of $f$ to $\bigoplus_{i=2}^{r} Y_{i}$ is epic. If $r>4$, then the lemma follows from Lemma 5. Assume that $r=4$ with all $Y_{i}$ non-projective. Note 
that $X$ is not projective by Corollary 4. By the dual of Lemma 5, we have $\ell(D \operatorname{Tr} X) \geq \ell(X)$ since $X$ has an injective successor.

Let $h: D \operatorname{Tr} X \rightarrow \bigoplus_{j=1}^{n} W_{j}$ be a source map with the $W_{j}$ indecomposable, and $W_{j}=D \operatorname{Tr} Y_{j}$ for $1 \leq j \leq 4$. Since the co-restriction of $f$ to $Y_{3} \oplus Y_{4}$ is epic, by Lemma 3 , the co-restriction of $h$ to $W_{j}$ with $j \neq 3,4$ is epic. Similarly considering separately the co-restrictions of $f$ to $Y_{2} \oplus Y_{4}$ and $Y_{2} \oplus Y_{3}$ which are epic, we deduce that the co-restrictions of $h$ to $W_{3}, W_{4}$ are epic. Therefore any sectional path in $\Gamma_{A}$ ending with $X$ contains no projective module by Corollary 2. In particular, $D \operatorname{Tr} Y_{i}$ is not projective for $1 \leq i \leq r$. Hence $D \operatorname{Tr} X$ has no projective predecessor and is not on any oriented cycle in $\Gamma_{A}$ by Lemma 5 . Therefore $X$ admits no projective predecessor in $\Gamma_{A}$. Moreover, $X$ is not on any oriented cycle in $\Gamma_{A}$ since $D \operatorname{Tr} X$ is not.

We are ready to get our main result.

Theorem 7. Let $A$ be an Artin algebra, and let

$$
0 \rightarrow X \stackrel{f}{\longrightarrow} \bigoplus_{i=1}^{r} Y_{i} \stackrel{g}{\longrightarrow} Z \rightarrow 0
$$

be an almost split sequence in $\bmod A$ with the $Y_{i}$ indecomposable. Assume that $X$ has a projective predecessor and $Z$ has an injective successor in $\Gamma_{A}$. Then $r \leq 4$, and $r=4$ implies that one of the $Y_{i}$ is both projective and injective, whereas the others are neither.

Proof. Let $r \geq 4$. We consider the first case where $\ell(Z) \geq \ell(X)$. Then by the dual of Lemma 5 , one of the $Y_{i}$ is injective. By Lemma 6 , we infer that $r=4$ and one of the $Y_{i}$ is projective. It is now easy to see that one of the $Y_{i}$ is both projective and injective, and the others are neither. A dual argument will show that the theorem holds in the case where $\ell(X) \geq \ell(Z)$.

R e m a r k. It is well-known that if $A$ is of finite representation type, then any indecomposable module has a projective predecessor and an injective successor in $\Gamma_{A}$. Hence the above result generalizes the Bautista-Brenner theorem [3].

Proposition 8. Let $A$ be an Artin algebra, and let $X$ be an indecomposable module in $\bmod A$ which is on an oriented cycle in $\Gamma_{A}$. If $f: X \rightarrow$ $\bigoplus_{i=1}^{r} Y_{i}$ is a source map with the $Y_{i}$ indecomposable then $r \leq 4$, and $r=4$ implies that one of the $Y_{i}$ is projective. Dually, if $g: \bigoplus_{j=1}^{t} Z_{j} \rightarrow X$ is a sink map with the $Z_{j}$ indecomposable then $t \leq 4$, and $t=4$ implies that one of the $Z_{j}$ is injective. 
Proof. Assume that $f: X \rightarrow \bigoplus_{i=1}^{r} Y_{i}$ is a source map with the $Y_{i}$ indecomposable and $r \geq 4$. Let

$$
X=X_{0} \rightarrow X_{1} \rightarrow \ldots \rightarrow X_{n-1} \rightarrow X_{n}=X
$$

be an oriented cycle in $\Gamma_{A}$ with $n \geq 2$. If there is a sectional path from $X$ to an injective module in $\Gamma_{A}$, then we are done by Lemma 6 .

Assume now that there is no sectional path from $X$ to an injective module in $\Gamma_{A}$. By a result of Bautista and Smalø [4], there is a minimal $m \leq t$ such that $X_{m}=\operatorname{Tr} D X_{m-2}$. Then $X_{j}$ is not injective for all $0 \leq j<m$. Thus $\operatorname{Tr} D X$ is also on an oriented cycle in $\Gamma_{A}$. If $\ell(\operatorname{Tr} D X)>\ell(X)$ then, by the dual of Lemma 5 , we infer that one of the $Y_{i}$ is injective, which is a contradiction. Hence $\ell(X) \geq \ell(\operatorname{Tr} D X)$. By Lemma 5 , one of the $Y_{i}$ is projective. Using now the dual of Lemma 6 , we deduce that $r=4$. The proof is complete.

Recall that if $X \rightarrow Y$ is an arrow in $\Gamma_{A}$, then its valuation $\left(d, d^{\prime}\right)$ is defined so that $d^{\prime}$ is the multiplicity of $X$ in the domain of the sink map for $Y$ and $d$ is the multiplicity of $Y$ in the codomain of the source map for $X$.

A path $X_{0} \rightarrow X_{1} \rightarrow \ldots \rightarrow X_{n-1} \rightarrow X_{n}$ in $\Gamma_{A}$ is said to be pre-sectional if $D \operatorname{Tr} X_{i+1}=X_{i-1}$ for some $0<i<n$ implies that the multiplicity of $X_{i-1}$ in the domain of the sink map for $X_{i}$ is greater than one [7].

Lemma 9. Let $X \rightarrow Y$ be an arrow in $\Gamma_{A}$ with valuation $\left(d, d^{\prime}\right)$. Assume that both $d$ and $d^{\prime}$ are greater than one. Then neither $X$ nor $Y$ is on an oriented cycle. Moreover, either $Y$ has no projective predecessor or $X$ has no injective successor in $\Gamma_{A}$.

Proof. Let $f: X \rightarrow Y$ be an irreducible map. First assume that $f$ is an epimorphism. Then $Y$ is not projective. Let $g: D \operatorname{Tr} Y \rightarrow X \oplus X_{1}$ be a source map. Then the co-restriction of $g$ to $X_{1}$ is an epimorphism. Note that $X$ is a summand of $X_{1}$ since $d^{\prime}>1$. The co-restriction $h$ of $g$ to $X$ is an epimorphism. By Corollary 2, any sectional path in $\Gamma_{A}$ ending with $Y$ contains no projective module. Since $d>1$ and there is an irreducible epimorphism $h: D \operatorname{Tr} Y \rightarrow X$, we similarly conclude that $X$ is not projective and there is an irreducible epimorphism $f_{1}: D \operatorname{Tr} X \rightarrow D \operatorname{Tr} Y$. Note that the valuation of the arrow $D \operatorname{Tr} X \rightarrow D \operatorname{Tr} Y$ is also $\left(d, d^{\prime}\right)$.

By induction we have $D \operatorname{Tr}^{n} Y \neq 0$ for all $n \geq 0$, and any sectional path in $\Gamma_{A}$ ending with $D \operatorname{Tr}^{n} Y$ contains no projective module. Therefore $Y$ has no projective predecessor in $\Gamma_{A}$. Now the arrow $X \rightarrow Y$ is contained in a left stable component of $\Gamma_{A}$, say $\Gamma$. For all $n>0$, there is a pre-sectional path

$$
D \operatorname{Tr}^{n} X \rightarrow D \operatorname{Tr}^{n} Y \rightarrow D \operatorname{Tr}^{n-1} X \rightarrow \ldots \rightarrow D \operatorname{Tr} Y \rightarrow X \rightarrow Y
$$

in $\Gamma_{A}$. Thus $Y$ is not $D$ Tr-periodic $[7,(1.16)]$. Therefore $\Gamma$ contains no 
oriented cycle since $X \rightarrow Y$ has non-trivial valuation $\left(d, d^{\prime}\right)[8,(2.3)]$. Thus $Y$ is not on any oriented cycle in $\Gamma_{A}$, and hence $X$ is not either. Dually, if $f$ is a monomorphism, then $X$ has no injective successor and neither $X$ nor $Y$ is on an oriented cycle in $\Gamma_{A}$.

Finally, we have the following.

Proposition 10. Let $A$ be an Artin algebra, and let $X \rightarrow Y$ be an arrow in $\Gamma_{A}$ with valuation $\left(d, d^{\prime}\right)$. If the arrow $X \rightarrow Y$ is on an oriented cycle in $\Gamma_{A}$, then $d d^{\prime} \leq 3$.

Proof. Suppose that the arrow $X \rightarrow Y$ is on an oriented cycle in $\Gamma_{A}$. Assume that $d \geq 4$. By Proposition 8, we infer that $d=4$ and there is a source map $f: X \rightarrow \bigoplus_{1}^{4} Y$ with $Y$ projective. Hence we have an almost split sequence

$$
0 \rightarrow X \stackrel{f}{\longrightarrow} \bigoplus_{1}^{4} Y \stackrel{g}{\longrightarrow} \operatorname{Tr} D X \rightarrow 0
$$

Since the co-restriction of $f$ to $\bigoplus_{1}^{3} Y$ is a monomorphism, so is the restriction of $g$ to $Y$. Hence by the dual of Corollary 2, we infer that any sectional path in $\Gamma_{A}$ starting with $X$ contains no injective module. Since $X$ is on an oriented cycle, using the Bautista-Smalø theorem, we deduce that $\operatorname{Tr} D X$ is also on an oriented cycle. Hence $Y$ is injective by Proposition 8, which is a contradiction. Thus $d \leq 3$. Dually $d^{\prime} \leq 3$. Moreover, by Lemma 9 , either $d=1$ or $d^{\prime}=1$. Therefore $d d^{\prime} \leq 3$.

\section{References}

[1] M. Auslander and I. Reiten, Representation theory of artin algebras III: Almost split sequences, Comm. Algebra 3 (1975), 239-294.

[2] - - - Representation theory of artin algebras $I V$ : Invariants given by almost split sequences, ibid. 5 (1977), 443-518.

[3] R. Bautista and S. Brenner, On the number of terms in the middle of an almost split sequence, in: Lecture Notes in Math. 903, Springer, Berlin, 1981, 1-8.

[4] R. Bautista and S. O. Smalø, Non-existent cycles, Comm. Algebra 11 (1983), $1755-1767$.

[5] D. Happel, U. Preiser and C. M. Ringel, Vinberg's characterization of Dynkin diagrams using subadditive functions with applications to $D$ Tr-periodic modules, in: Lecture Notes in Math. 832, Springer, Berlin, 1980, 280-294.

[6] M. Harad a and Y. Sai, On categories of indecomposable modules, Osaka J. Math. 7 (1970), 323-344.

[7] S. Liu, Degrees of irreducible maps and the shapes of Auslander-Reiten quivers, J. London Math. Soc. (2) 45 (1992), 32-54.

[8] -, Semi-stable components of an Auslander-Reiten quiver, ibid. 47 (1993), 405416.

[9] -, On short cycles in a module category, preprint. 
[10] I. Reiten, The use of almost split sequences in the representation theory of artin algebras, in: Lecture Notes in Math. 944, Springer, Berlin, 1982, 29-104.

[11] C. M. Ringel, Tame Algebras and Integral Quadratic Forms, Lecture Notes in Math. 1099, Springer, Berlin, 1984.

[12] Y. Zhang, The structure of stable components, Canad. J. Math. 43 (1991), 652672 .

DEPARTMENT OF MATHEMATICS

NATIONAL UNIVERSITY OF SINGAPORE

SINGAPORE 0511

REPUBLIC OF SINGAPORE

Received 28 January 1993;

in revised form 30 April 1993 